\title{
Programming Visual Representations. Evolutions of Visual Identities between Tangible and Intangible
}

\author{
Guida, Francesco Ermanno ${ }^{a}$ \& Voltaggio, Ernesto ${ }^{b}$ \\ åDipartimento di Design. Politecnico di Milano, Italy. francesco.guida@polimi.it. \\ bopenDot Community, Italy. erni.volt@gmail.com.
}

\begin{abstract}
The communication design field it is considerably changed in the last 20 years and more as well as the role of the designer. Technology has modified the daily work tools, and new possible relations between the designer, the commitment, and the final user can be underlined.

Observing some of the most experimental practices, new visual languages have drawn the attention, affected by innovative approaches and mixed competencies. The area of visual identities is especially of interest, not excluding other areas of experimentations.

The phenomenon of the so-called dynamic or post-logo identities underlined the possibilities of using more fluid and expressive, variable, context related, processual, performative, non-linear, consistent visual languages instead of the usual and static repetition of a logo or an imposed series of rules (Felsing, 2010). However, also their contradictions in making recognizable an organization and in the visual identity daily management.
\end{abstract}

An interesting evolution to be underlined is in the use of the digital tools, not anymore in a passive way but in an active way. Visual designers can build their digital tools basing them on design and esthetic needs. The designer is not anymore just the user of ready-made digital tools, becoming himself programmer of customized digital toolboxes by using open source codes or hardware like Arduino. Not just a DIY attitude but something that it is changing the control knobs of a design system in all its process and development.

As far as technology support is relevant, technical matters are relegated in the background on behalf of abstraction and data parametrization that means on behalf of a meta-design level. The use of programming in creative and visual communication design processes "empowers the designer, freeing he from the constraints of predefined computational tools, and promoting creative freedom in the construction of visual metaphors" (Duro, Machado, Rebelo, 2012). The aim of this paper is to argue this recent evolution in the field of visual identities and the wider area of communication design practices.

Keywords: Communication design, visual identity, brand identity, post-logo. 


\section{Visual Identities: from static to dynamic systems}

In the broad context of visual communication design disciplines, talking about "identity" usually refers to the field of the image. It refers to the representation of an organization, of a subject (the "personality"), through the use of a particular visual code organized on the basis of a predefined grammar that establishes the rules of operation and combination of primary (symbol or/and logotype), subsidiaries elements (typeface, colors, any other visual elements to be used as coordination elements) and applications (the socalled "points of contact"). Hence the definition of corporate identity, whose normative expression is the "manual", the "rulebook", useful to avoid dispersion and weakening of the communication; a typical phenomenon of entities with different interests and characterized by significant fragmentation.

By the use of the term "image", we may refer to something intangible that marks and allows to identify a subject, making it tangible, so that it can coincide with the term "identity". The expressions of "visual identity" and "corporate identity" thus tend to coincide. It is necessary to specify that "corporate identity" can allude to something wider than just the visual identity plan. Something that today we call "brand identity", implying multi-dimensional and multi-channel scales.

Henrion and Parkin (1967) define "corporate image" as "the totality of pictures or ideas or reputations" of a "personality" (single entity or organization that is) in the mind of the people who interact with such "personality". This idea is formed over time through a series of "points of contact", such as buildings, products, packaging, printing, vehicles, publications, uniforms, promotional activities, etc. In other words through all that complex of actions, channels, and tools that enable users or clients to get in touch with an organization.

The points of contact - at least those who need a "visual interface" - is precisely the object of visual communication projects, while also involving other design skills depending on the application, the scale, the size that they will have to take. The use of the "manual", initially utilized in the supra-national dimension organizations (prototypical case, among many, is that of Shell) and then adopted in all areas in which it was requested a project of visual identity, it resulted in the production of corporate images frequently monolithic, based on the logo and its replication on the various applications, without significant variations. However, according to this model, firmly based on an atomistic and structuralistic approach, an "image" is not able to exhaust all the dimensions of a "personality". In the sixties had already spread the idea, inspired by systemic thinking, that "[...] for the receiver, image rests on a substrate consisting of an assemblage of objects. These objects, in the perception function like the constitutive features of a unitary complex of signs" (Anceschi, 1988). This "unitary complex of signs" is, in fact, the system of points of contact outlining the traits of this "artificial person" (e.g., an organization).

In the culture of "corporate image", "corporate personality" (or "artificial person") is a figure (or person) that "consists of objects" and corporate image is communication through a juxtaposition of objects, or rather narration through objects. The corporate image then produces an "image", the portrait of the organization's artificial person, through the control of the appearance of individual objects. Where control is employed through a legislative system of implementation and combination of rules (the "Manual"), that organizes grammar and syntax of visual communication, with the limitations that bureaucratic or technocratic application of the rule may have.

The systemic approach to corporate image project is clearly manifested when switching from application of the rule to the program, or if you agree to the combinations of the elements, "meetings between formula and circumstance", able to surprise beyond normative forecasts, passing by the size of appearance (image) to the behavior. Henrion and Parkin (1967) about it spoke of "design coordination", to emphasize the importance of designers directing role in defining an identity. 
In the latest twenty years, the idea that an identity centered on a logo could be the only and most effective possibility has been discussed in various practices and some specialized literature. A "post-logo" concept has emerged emphasizing the possibility to express effectively a "personality" using criteria which underline differences, or inconsistencies, according to a most dynamic and flexible logic. Visual identity tends to represent the varieties of a "personality", giving greater importance to the previously considered subsidiary elements and their combination on the basis of criteria that always allow the recognition of the communicating subject using a system of rules that takes account of its complexity, its inherent differences, the variety of possible expressions (Felsing, 2010; Van Nes, 2013).
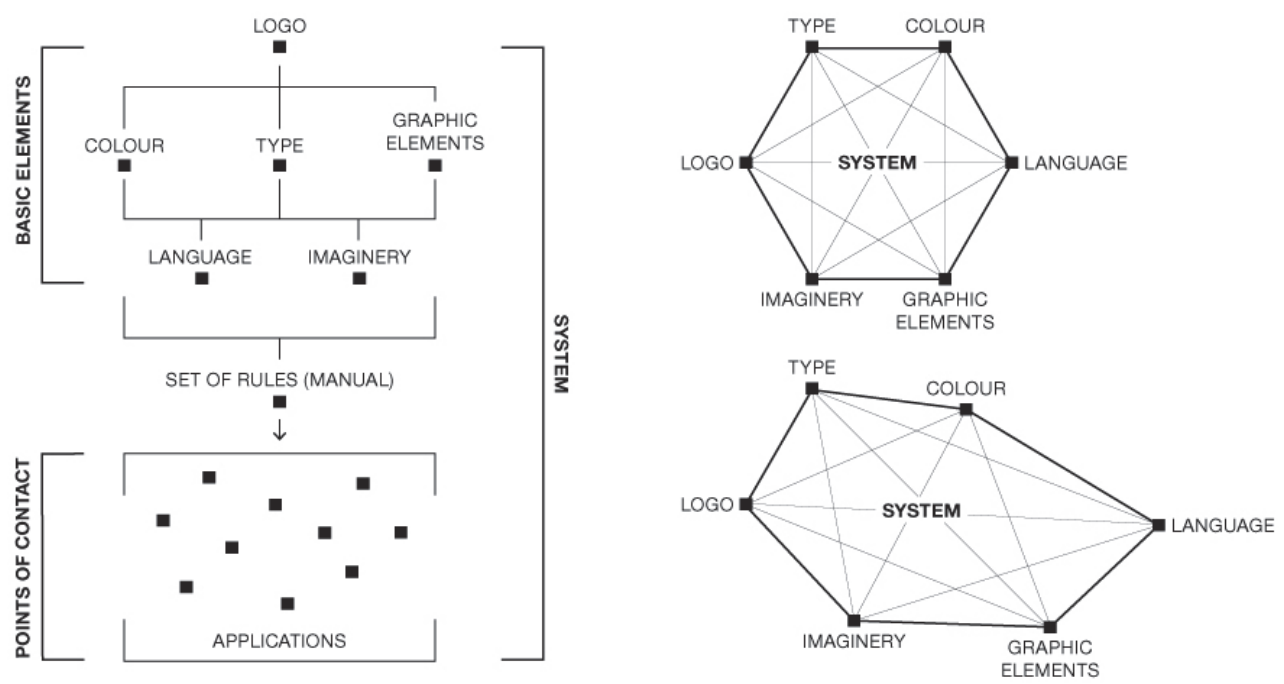

Fig. 1 Left, a representation of the typical visual identity structure (made by the authors). On the right it is represented how dynamic or flexible visual identities work. Source: Van Nes, (2013).

The definition of "identity" it is extremely interesting itself: it is possible to define it as a "fundamental unity that is achieved through the multiplicity of aspects." So at the base there is a unit, equality between different elements, however, belonging to the same set, to the same context. The identity of a subject, its unity, it is reinforced by the differences of its attributes and its modes of expression.

While there are the organization's needs to set up new relationships with its stakeholders in new ways, on the other hand, there are trends of design thinking and approach that similarly tend to innovation: "a brand is no longer simply a nice, clean logo that is attached in the same place every time. A brand is a platform, a brand is flexible, a brand is a place for exchange, it is not fixed, and therefore there is no such thing as a single brand. Methods exist which allow a shape to form, which allow communication and recognizable behavior, but this is no longer about something inflexible and permanent" (Shaughnessy, 2008).

This attitude has resulted in a direction in which the project of "corporate branding" (evolution of the culture linked to the one of "corporate identity" of the sixties) waives the imposition of trivial rules (or trivializing) and dictates the long sweltering, tending more towards fluid and expressive languages. Some organizations developing structures and communication tools have understood that it could be more 
efficient to modulate the tone of their communication, adapting to the variety of the target, rather than fall from a single, monotonous voice. If a personality in general terms is not possible to define it as a monodimensional subject, it surely can not be an organization and its resulting communication strategy, which moreover is aimed at markets that are definitely multi-dimensional (Dorrestejin, 2007).

One of the factors that contributed to this new scenario it is certainly the growth of the digital environment. In the last 20 years, the system of visual identities has been revitalized by the adoption of formal building tools and processes nearer to meta-design matrices. That is a change from closed (e.g. logo-centered) to open systems. Particularly of interest is the use of programming codes to generate and manage visual artifacts on a parametric basis.

\section{The use of programming in visual design}

The use of programming in visual design it is not something peculiar only to the so-called Generation $Y$ (those born between 1980 and 1996) designers, for which the use of technology is natural if not obvious (Wicht, 2011). In visual and graphic design there is a particular field that emerged in the last 30 years as extremely suitable to experimentations in this direction. Type designers are used to comparing their designs both to formal and technical meanings. Moreover, because of this inner nature of the design of types, when digital technology arrived in the 1980s, "type designers made the accidental a starting point for new ideas. Some, as they became more adept with the new technology, began to intervene in the underlying computer code" (Crow, 2008).

Experiments like the ones promoted by Fuse or Emigre magazines (that became a type-foundry too) opened to commercial practices new perspectives in the use of computer and enhanced several critical considerations. The most evident result of those conditions it can be found in the Dutch group called Letterror, which enthusiastically pioneered the adoption of coding as a design tool, with their typeface Beowolf in 1991. They were the first designers to use code to randomize typography and their aesthetic undoubtedly celebrated the handmade and the physical. In a certain way, Letterror harnessing digital technology to create letterforms by art-directing the multiple possibilities programmed into their bespoke software engines (Crow, 2008), anticipated most recent formal and procedural issues. Issues that were already experimented by computer scientist and mathematician Donald Knuth in the 70s. Knoth developed Metafont, a program that worked with the idea of parametrical fonts and would allow designing an infinite number of typefaces (Knoth, 2011).

If type design appears to be a suitable field of experimentation, in many other areas of visual design, such visual identity, it is possible to find interesting evolutions. Also, it is useful to mention just a few cases acquired from the professional practice. 


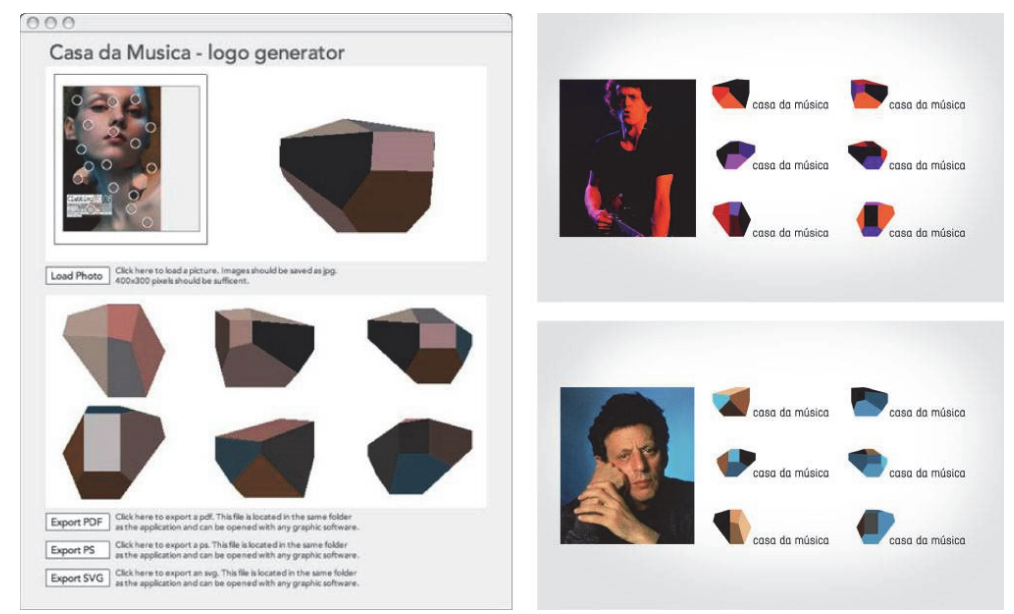

Fig. 2 Logo generator - developed using Processing - for the Casa da Musica. Oporto (Portugal), design Stefan Sagmeister, (2007).

Chronologically the logo-generator for the Casa da Musica (Sagmeister, 2007; fig. 2) is ones of the very first. It works on six versions of a basic sign, necessarily inspired by the Rem Koolhaas's design of the building, showed from different perspectives. Through the different views of the building, 17 facets are defined - from those a 17-point color-picking mechanism is created as customized software. That allows to select from images the colors of the sign to be used in the various communication matter for particular events or the staff (e.g. business cards). The logo changes in every application and media and the colors change too. The software offers an endless variety of solutions both for the audience and for those who have to manage the identity every day.

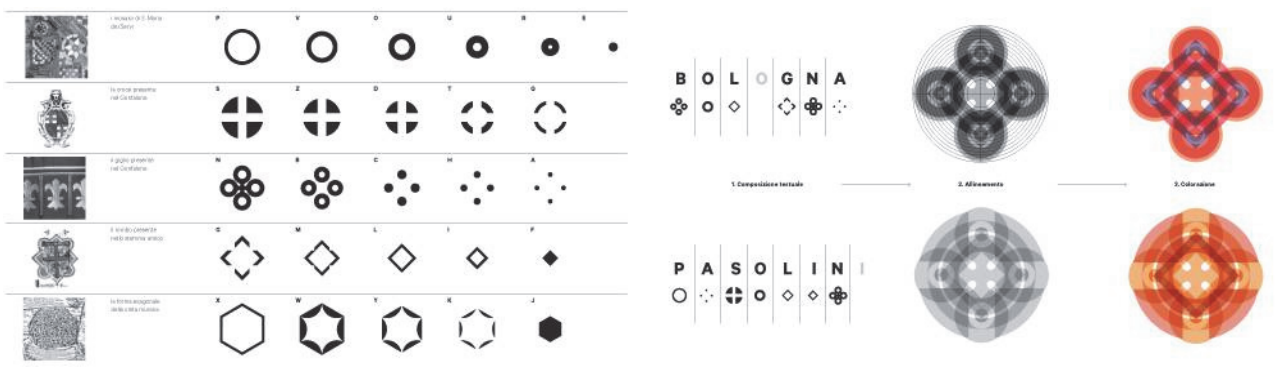

Fig. 3 Basic elements of the visual alphabet and set of rules for logo arrangement, "è Bologna” city brand, design Matteo Bartoli and Michele Pastore, (2013).

Another case study to mention is the one of the place brand for Bologna, adopted in 2013. The visual system (designed by Matteo Bartoli and Michele Pastore; fig. 3) is based on a visual alphabet in which the single letters are replaced by geometric abstract signs inspired to a typical Italian historical imaginary. In this way, the richness of elements and tangible and intangible values -that can be typically referred to an Italian town- are translated. The forms freely take up again some figurative archetypes of the Italian city and more precisely of Bologna (Vit, 2014), such as walls, brick mosaic, the lily and the heraldic cross. Through a customized online software (available at http://ebologna.it/) it is possible for everyone to write what "Bologna is" ("è Bologna"). This is not just an adaptive and flexible visual system, but participatory 
too (Vit, 2014). Another point that emerges is that of a brand as a platform and of a logo conceived as a people's heritage, which belongs effectively to everybody.
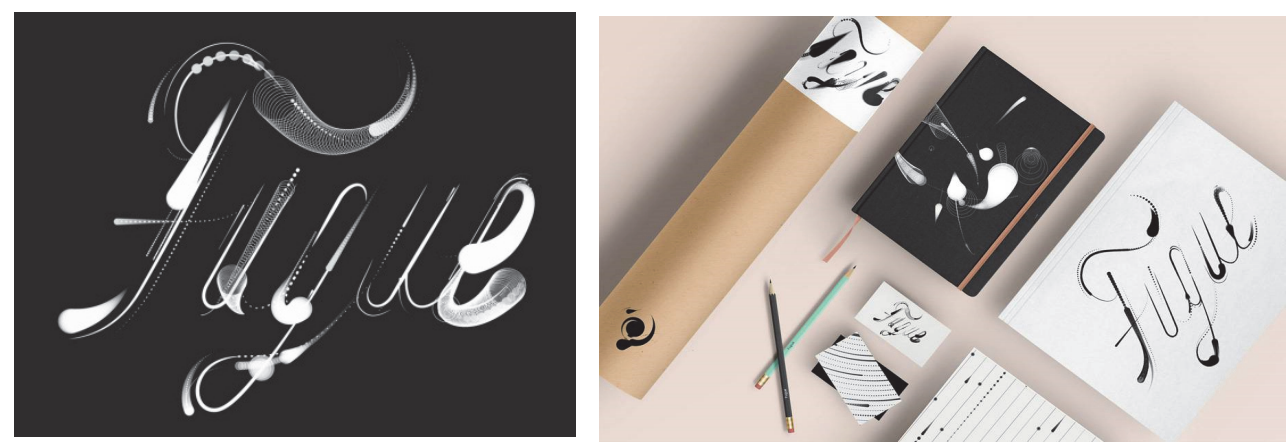

Fig. 4 Fugue identity, logo detail and some applications, design Stefan Sagmeister and Jessica Walsh, (2015).

The last example to mention is the one of Fugue (Sagmeister and Walsh, 2015; fig 4), a company specialized in the automation of the creation, operations, and regeneration of cloud infrastructure. The brand concept visualizes the ephemerality and embodied core attributes of lineage and elegance. The logo works like the software does: it constantly regenerates itself while data moves from one point to another. Since the company is about constant regeneration and evolution, the design agency developed an application that allows them to import any SVG file so to easily create new patterns and illustrations over time as needed. The user can then alter the size, speed and density with the application to increase clarity or create specific styles of animations based on their need and export tifs or .mov files. The logo application also has a drawing function so that you can draw abstract visuals in the Fugue language using a tablet device.

What emerges is the use of code to program devices to produce and generate visual artifacts. Traditionally, when graphic designers have to design a logo or a visual identity system, they reduce contents and values of an organization through signs by using professional tools. In the past those were pencils, photos, scissors and so on, then the very first photo and layout software. The use of code opens to the possibility to create devices (toolboxes, like logo-generators are) to produce and generate visual artifacts able to manage variations. Visual designers have to define still the set of rules and a framework to shape a visual identity. So the designer part becomes the one who sets parameters to generate forms not losing sight of a visual identity system main task that is to identify and to make recognizable an organization (Téllez Bohórquez, 2011).

\section{The designer-developer}

The use of proprietary software (or closed source software) that limit the possibilities to the ones provided by package developers did not result until today in significant innovations in terms of visual language. Those limitations have been effectively underlined by the expression "form follows software" (Serriano, 2003), which make evidence to the need, by the creative world people, to experiment all open source programming codes possibilities. Reas et al. (2010) expressed well this way of thinking: "Proprietary software products are general tools designed for the production of specific types of forms (...). To go beyond these limitations, it is necessary to customize existing applications through programming or to write your own software". A direction that Galanter (2003) already summarized, therefore: "It was 
seemingly inevitable that soon after the adoption of the computer by designers as a manual tool for CAD, there would follow the adoption of genetically inspired algorithms for the creation and selection of variations".

The use of codes makes possible to manage and - according to Manovich (2001) - generate multiple formal solutions, to set up automation, repetition, scalability and variation processes. Those systems can be considered as facilitation tools of a creative act, as they allow to spend more time in experimentation, research, production; and the final result can be more satisfying for the designer as well as consistent with the design ambitions and requests (Reas et al., 2010).

The visual designer knowledge, way of thinking and workflow are changing because of the new possibilities offered by programming (Lehni, 2011). Moreover, a new practitioner is emerging: the designer-programmer or the designer-developer. A practitioner who has no fear in using the daily work tools, as computers are, in a more consistent way. Computational design usually requires the designer to write programs and because of that, it is possible to mistake the practice of computational design as a technical skill rather than a way of thinking. Learning to program and engage the computer more directly with code, opens the possibility of not only creating tools, but also systems, environments, and entirely new modes of expression. It is here that, using McLuhan metaphor, the computer ceases to be a tool and instead becomes a medium (Reas et al., 2010).

Moreover, it is to consider, accessibility to instructions and information related to codes, allowed by the global open source culture, as a critical component in this evolutionary process (Lehni, 2011). This culture allows sharing knowledge, results as well as codes, making possible a constant upgrade. A knowledge available for all, blurring the borders of a merely professional disciplinary field.

\section{Experimental outputs}

Consistently to those critical issues and practices, a series of experimental projects have been developed in the Visual Communication Design Final Synthesis Studio of the Communication Design Bachelor at Politecnico di Milano over the last three years.

The brief was to design visual identities programming and using open source codes and, when possible, hardware like Arduino or Genuino so to experience a multidisciplinary approach and test more contemporary design methods and processes.

Unreal organizations were assumed as subjects of the design, this to allow students to work deeper on the conceptual side and to look for case-histories (to be assumed as inspirations) not necessarily referred to the assigned organization. As well as to get students used to cross disciplinary borders and to criticize fixed fields. Triggs (2003) expression on experimentation as a way to find solutions, even in areas that we (as teachers and/or practitioners) or students do not precisely know, it is incredibly fitting. A "learning by doing" approach, aimed to result in prototypes, is the main methodological framework. During projects development, students experienced something close to the definition of "thinkering" (think + tinkering) given by Paola Antonelli (2011) in which a final result it is possible through progressive collective refinements.

The applied methodology can be summarized as in the following schema (fig. 5), inspired to the spiral model of software development by Barry Boehm (Dubberly, 2005). This model perfectly representes repeating cycles of design with a spiral path moving away from a center starting point. In each of the 4 main phases (Objective setting; Risk assessment and reduction; Development and validation; Planning the 
next phases) students experienced different design steps approaching gradually and practicaly their final solutions. The model seems to fit well to the adopted laboratorial design process.

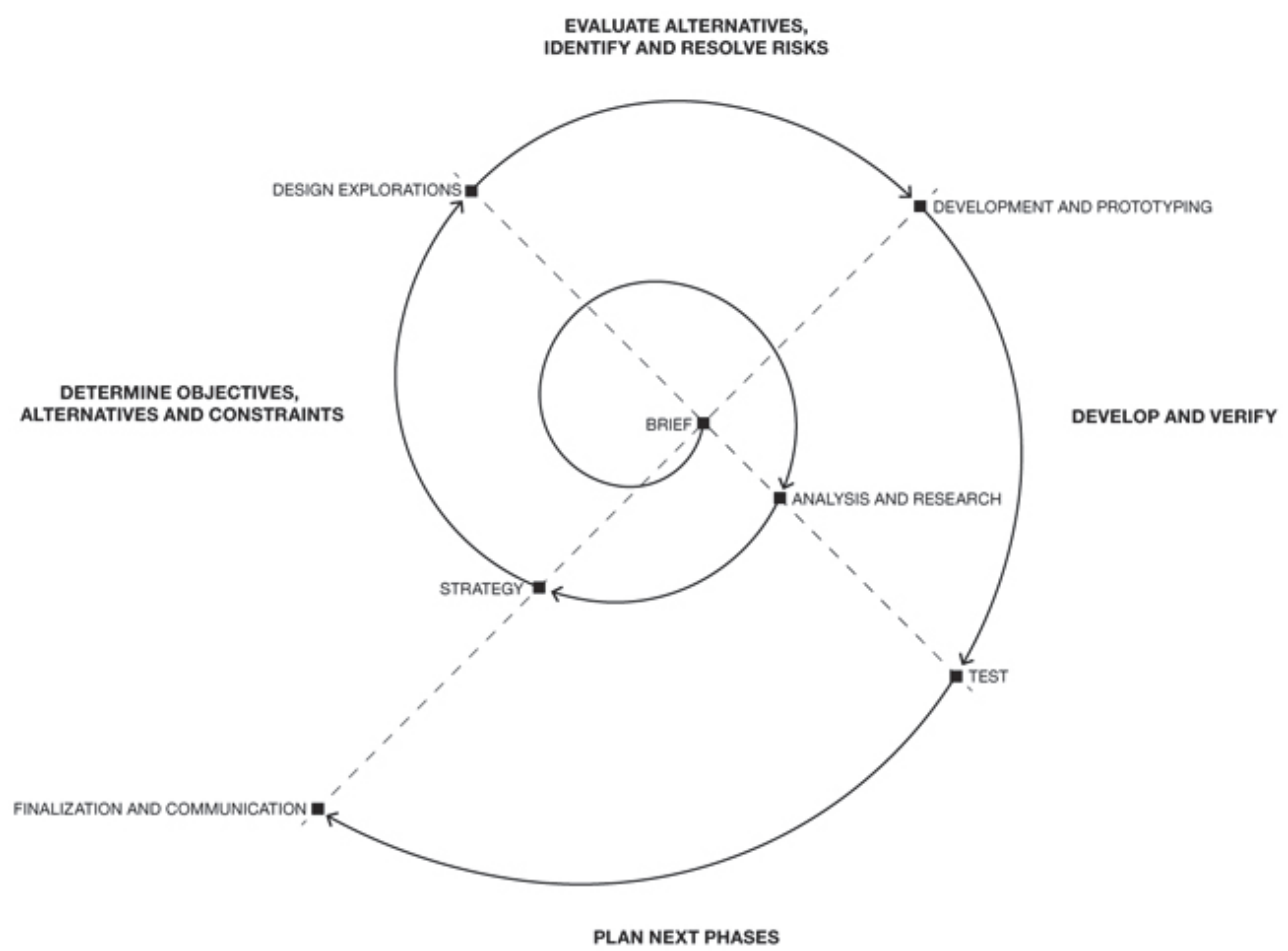

Fig. 5 The spiral model of the methodology applied among years in the experimental Design Studio at Politecnico di Milano.

Every year the class was organized in groups of 4 or 5 students each. First, each group has to define the whole concept and the organization of the assigned subject, his aims, his values through targeted research (intangible issues); then to design the visual system, defining appropriate communication channels, tools and applications (tangible issues).

During the first year of this experimental Design Studio (2013-14) each group had a specific subject to work on (e.g. nation branding, sports event, currency system, political party, and so on). Each group had to develop his project defining parameters and rules of the visual identity variations to be programmed by using the code VVVV. The following example it is to be assumed as peculiar of this design experimentation.

The visual identity for an invented hacker micronation located in the Westman Islands - a series of small islands sited south of Iceland -, is mainly based on two elements: the Vegvisir (the ancient Icelandic magical stave intended to help the bearer find their way through rough weather) inspired an alphabet; the glitch (the bitmap images failure) inspired the graphic elements of coordination. The Vegvisir-inspired elements are used to arrange the primary symbol of the nation and to write customized codes to use as customized fiscal codes. 

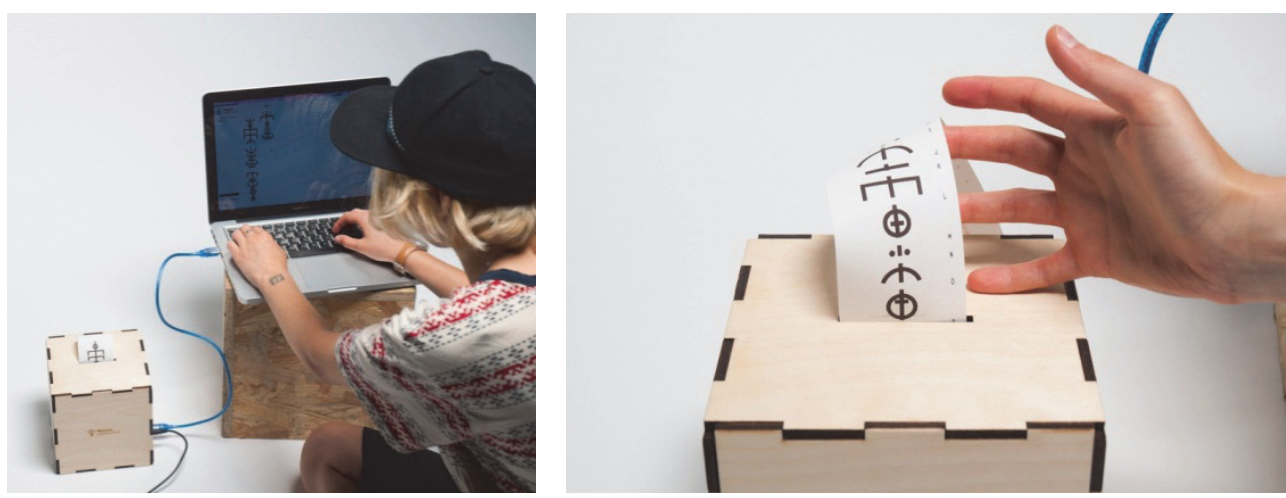

Fig. 6 On-screen writer and printer for the Westman Islands nation's brand identity.. Design by Mariagloria Posani, (2014).

A particular toolbox has been designed and prototyped to write and print the visual code (fig. 6). The toolbox is composed of an on-screen writer called Muninn and of a printer named Huginn (the two ravens that fly all over the world and bring information to the god Odin). The glitch inspired elements are used in two ways. First, to arrange the visuals of printed and digital applications. Also, as a digital nation's flag that is shaped through a particular sound-reactive generator: two central rectangles change on sound volume and

tone variations; a series of disturbing pixels increase and arrange themselves following the ground noise. The overall elements give a visual representation of participatory democracy.
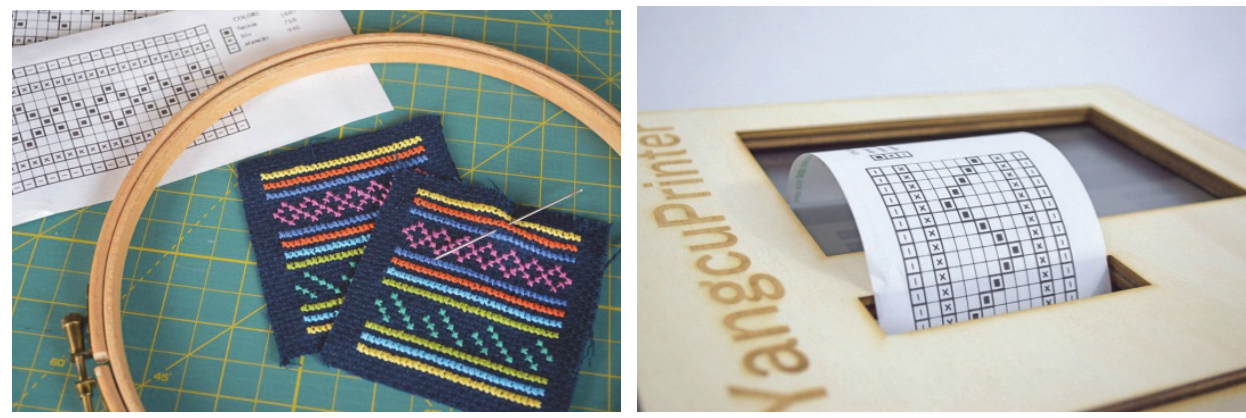

Fig. 7 Yangcu Identity points of contact and the cross-stitch printer. Design by Daniela Arienti, Nicolas Attolico, Andrea Benedetti and Clelia Codella.

The following year (2014-15) the class worked on visual identities referred to unrecognized nations inscribed in the list of the UNPO (the Unrepresented Nations and People Organization). The aim was to stimulate considerations on cultural expressions to be used as soft-power elements and objects of communicational pression to be recognized.

One of those results was referred to the people of Degar-Montagnard (fig. 7), located in the Vietnamese region, in South East Asia. They are called "mountain people". Starting from textile compositions, the group developed a texture based visual identity and a typographic system as basic elements of a communication system called Yangcu. To empower the communication, a toolbox has been realized. This 
allows to print textures as cross-stitch masks and, at the same time, to easily share cultural elements by the customization of a daily object.
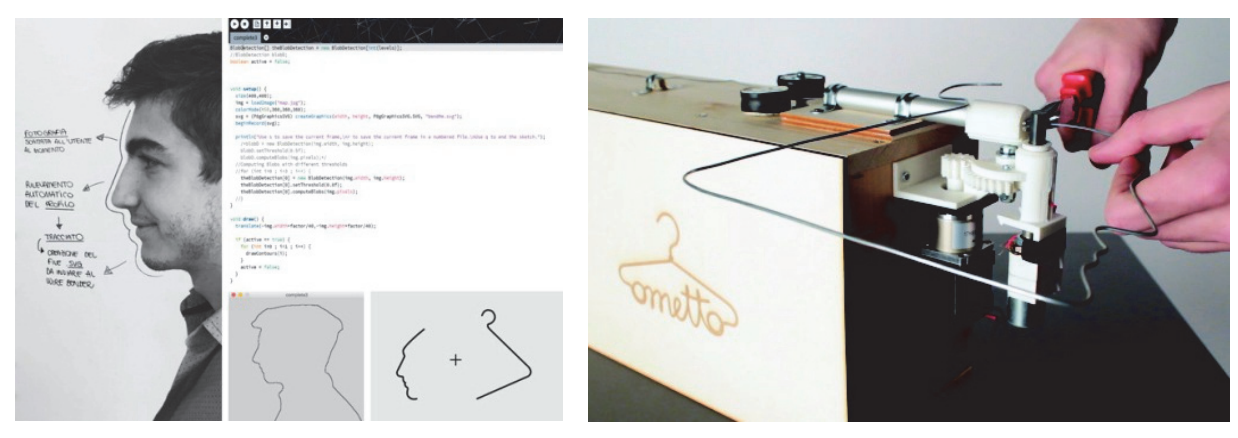

Fig. 8 Ometto identity: the contour tracking schema and the Wire Bender. Design by Martina Bonanomi, Gianluca Bruno, Luana Caiazzo, Tommaso Diena, Valeria Esposti.

During last year (2015-16) the class worked on visual identities referred to unreal companies producing everyday objects like umbrellas, light-bulbs, buttons or hangers. Each group has to design visual identity in both two and three dimensions. Furthermore, studends had to work on the design of an experience to be contextualized in a fixed area of $4 \times 4$ meters, by realizing devices ("communication machines") that interpreted companies' values making those accessible to users. In the case of a hangers producer (called Ometto, that is an italian name for clothes hangers; fig. 8), a clean and minimalist brand identity was developed as well as a cutomized device. The device has two main sections: a contour tracking of the user's profile (programmed in Processing) and a wire bender (a revised version of DiWire Bender by PensaLab basicly developed with Arduino). The contour tracking program reads the svg file (of the user's), set the size of the product and sends the exact coordinates to the Wire Bender, which personalize a hanger with the user's profile.

\section{Conclusions}

The examples as mentioned above (both case studies and experimental outputs obtained with research through teaching) allow making some final considerations.

The real evolution that emerged in the field of visual identity design is in the use of the digital tools, not anymore in a passive way but in an active way. Visual and graphic designers can build their digital tools basing them on design and aesthetic needs. Interpretation of intangible issues (the ones of an organization to communicate as a brand) can be empowered by the use of intangible (codes) and tangible (hardware) tools. Results are still points of contact and experiences (tangible items), but innovation is in the creative process, instead of in the final result (Galanter, 2003), is in the "way to live our own creativeness" (Soddu, 1998). The above mentioned new approach to brand identity design is "open, unpredictable, functioning like an organism that modifies and adjusts itself to suit different contexts. Now, the designers are not aiming to make the audiences' mind to be imprinted with a logo, but aiming to create live dialogues with them" (Graphic, 2010).

However, the full potential of the computer as a programmable device to be used in the representation of organizations and the managing of a visual identity system remains still to be entirely explored. Applying 
programming in the creation of visual identity systems should make possible to redefine the idea of the manual (to be intended as the rulebook of a visual identity system) and the way the visual governance of a brand could work. Digital codes allow managing in a more flexible and practical way how a visual identity works in his everyday life.

It is clear that the role of the visual designer itself is changed. The line between "designer" and "developer" is apparently blurred, and this is not limited to screen or projected image (Reas et al., 2010). It also affects the design of physical spaces and experiencial spheres.

Finally, introducing those issues in a didactic context, emerged that programming environments and languages are never static, just like spoken languages that evolve during time. Programming evolves and is becoming more and more part of the designer toolset and open source is a key component in this evolutionary process. This way of working and designing should be encouraged, especially during students education so to make possible the use of digital tools in a more consistent and suitable way (Lehni, 2011).

\section{Acknowkedgements}

The authors would like to thanks the teachers and colleagues who worked during the last three years at the Communication Design Studio, Design School of Politecnico di Milano: Andrea Braccaloni, Pietro Buffa, Alessandro Masserdotti and the assistants Ambhika Samsen, Andrea Novali, Francesca Bozzia and Dario Verrengia. With them it has been possible to launch and share an actual, not easy and inventive method and design practice. Consequently, a grateful thought is for all students who accepted the challenge and enthusiastically followed his development.

\section{References}

ANCESCHI, G. (1988). Monogrammi e figure. Firenze: La casa Usher.

ANTONELLI, P. (2011). "States of Design 03: Thinkering". Domus, n. 948, June. $<$ http://www.domusweb.it/it/design/2011/07/04/states-of-design-03-thinkering.html $>$ [See: May 10, 2016].

CROW, D. (2008). "Magic box: craft and the computer" in Eye Magazine, vol. 18, n. 70. $<$ http://www.eyemagazine.com/feature/article/magic-box-craft-and-the-computer> [See: May 10, 2016].

DORRESTEIJN, T. (2009). Visual Branding. Amsterdam.

DUBBERLY, H. (2005). How do you design? A compendium of models. San Francisco: Dubberly Design Office. $<$ http://www.dubberly.com/wp-content/uploads/2008/06/ddo_designprocess.pdf $>$ [See: May 11, 2016]

DURO, L. and MACHADO, P. and REBELO, A. (2012). "Graphic narratives: generative book covers" in $A C M$ Siggraph Proceedings. Los Angeles, California.

FELSING, U. (2010). Dynamic identities in cultural and public contexts. Baden: Lars Muller Publishers.

GALANTER, P. (2003). "What is Generative Art ? Complexity Theory as a Context for Art Theory" in Proceedings of the GA2003 - 6th Generative Art Conference. Milan.

GRAPHIC (2010), Visual Identity Issue, n. 13. Editorial. Seoul: Propaganda Press. $<$ http://graphicmag.kr/index.php?/issues/13-visual-identity-issue/> [See: May 10, 2016].

HENRION, FHK and PARKIN, A. (1967). Design coordination and corporate image. London: Studio Vista.

KNOTH, C. (2011). Computed type design. MA Type Design thesis. Lausanne: ECAL / École Cantonale d'Art. $<$ http://christoph-knoth.com/shared/christoph_knoth___computed_type_-_theory_35.pdf $>$ [See: May 9, 2016]. 
Programming visual representations. Evolutions of visual identities between tangible and intangible.

LEHNI, J. (2011). "Teaching in the spaces between code and design" in Eye Magazine, no. 81, vol. 20. $<\mathrm{http} / / /$ www.eyemagazine.com/feature/article/teaching-in-the-spaces-between-code-and-design $>$ [See: May 10, 2016].

MANOVICH, L. (2001). The Language of New Media. Cambridge (MA): Mit Press.

REAS, C. and MCWILLIAMS, C. and BARENDSE, J. (2010). Form+code in design, art and architecture. New York: Princeton Architectural Press.

SERRIANO, P. (2003). "Form follows Software. Connecting >> Crossroads of Digital Discourse" in Proceedings of the 2003 Annual Conference of the Association for Computer Aided Design in Architecture. Indianapolis, Indiana.

SHAUGHNESSY, A. (2008). "Wolff Olins' intuitive branding” in Business Week, February 6. $<$ http://www.bloomberg.com/news/articles/2008-02-06/wolff-olins-intuitive-brandingbusinessweek-business-newsstock-market-and-financial-advice> [9 May 2016].

SAGMEISTER, S. (2007). Casa da Musica Identity. <http://www.sagmeisterwalsh.com/work/project/casa-damusica-identity/> [See: May 8, 2016].

SAGMEISTER, S. and WALSH, J. (2015). Fugue Identity. <http://www.sagmeisterwalsh.com/work/project/fugueidentity/> [See: May 8, 2016].

SODDU, C. (1998). "Introduction" in Proceedings of the GA1998 - 1st Generative Art Conference. Milan.

TÉLLEZ BOHÓRQUEZ, A. (2011). "Computación e identidad visual corporativa” in Proceedings of the XV Congreso Sigradi. Santa Fe, Argentina.

TRIGGS, T. (2003). The typographic experiment: radical innovation in contemporary type design. London: Thames \& Hudson.

VAN NES, I. (2013). Dynamic Identities. How to create a living brand. Amsterdam: Bis Publisher.

VIT, A. (2014). "Bologna is as Bologna does" in Underconsideration Brand New, February 13. $<\mathrm{http} / / /$ www.underconsideration.com/brandnew/archives/new_logo_and_identity_for_city_of_bologna_by_matteo_b artoli_and_michele_pastore.php\#.VzuGOpOLSRt> [See: May 9, 2016].

WICHT, P. (2011). "Generative Identity" in Enigma, July 21. <http://enigmaprod.ch/en/where-we-stand-3/identitegenerative/> [See: May 9, 2016]. 